\title{
A Platform for Studying Molecular and Cellular Mechanisms of Parkinson's Disease Based on Human Induced Pluripotent Stem Cells
}

\author{
DOI: $10.17691 / \mathrm{stm} 2016.8 .4 .20$
}

Received May 3, 2016

E.V. Novosadova, PhD, Researcher, Laboratory of Molecular Genetics of Somatic Cells :

E.D. Nekrasov, PhD, Researcher, Department of Genetic Basics of Cell Technologies;

I.V. Chestkov, Researcher, Department of Genetic Basics of Cell Technologies2;

A.V. Surdina, PhD, Senior Researcher, Department of Genetic Basics of Cell Technologies2;

E.M. Vasina, PhD, Senior Researcher, Department of Genetic Basics of Cell Technologies;

A.N. Bogomazova, PhD, Senior Researcher, Department of Genetic Basics of Cell Technologies;

E.S. Manuilova, PhD, Senior Researcher, Laboratory of Molecular Genetics of Somatic Cells ${ }^{1}$;

E.L. Arsenyeva, PhD, Researcher, Laboratory of Molecular Genetics of Somatic Cells ${ }^{1}$;

V.V. Simonova, Researcher, Laboratory of Experimental Neurocytology ${ }^{3}$;

E.V. Konovalova, PhD, Researcher, Laboratory of Experimental Neurocytology3;

E.Yu. Fedotova, MD, PhD, Senior Researcher, Department of Neurogenetics;

N.Yu. Abramycheva, PhD, Senior Researcher, Department of Neurogenetics3;

L.G. Khaspekov, DSc, Head of the Laboratory of Experimental Neurocytology3;

I.A. Grivennikov, DSc, Professor, Head of the Laboratory of Molecular Genetics of Somatic Cells';

V.Z. Tarantul, DSc, Professor, Deputy Director for Science';

S.L. Kiselev, DSc, Professor, Head of the Department of Genetic Basics of Cell Technologies;

S.N. Illarioshkin, MD, DSc, Professor, Corresponding Member of the Russian Academy of Sciences,

Deputy Director for Science, Head of the Brain Research Department ${ }^{3}$

${ }^{1}$ Institute of Molecular Genetics of the Russian Academy of Sciences, 2 Academician Kurchatov Square, Moscow, 123182, Russian Federation;

${ }^{2}$ N.I. Vavilov Institute of General Genetics of the Russian Academy of Sciences, 3 Gubkin St., Moscow, 119991, Russian Federation;

${ }^{3}$ Research Center of Neurology, 80 Volokolamskoye Shosse, Moscow, 125367, Russian Federation

The aim of the study was to obtain induced pluripotent stem cells (iPSCs) from patients with various forms of Parkinson's disease (PD), and to create on this basis a platform for studying the pathogenesis of the disease at the molecular and cellular level with the development of a protocol of the stem cell differentiation.

Materials and Methods. iPSCs were derived from cultured skin fibroblasts, taken from five patients with various forms of PD (PARK8, PARK2, GBA-associated and sporadic forms), and reprogrammed with the help of lentiviral vectors and on the basis of Sendai virus. The obtained iPSCs clones were cultured to the stage of embryonic bodies and, after spontaneous differentiation, stained immunocytochemically. Gene expression and neural markers in these iPSCs lines were analysed using reverse transcription polymerase chain reaction.

Results. The obtained iPSCs clones had a normal 46 XY karyotype, stained specifically with Oct4, Nanog, TRA-1-81 and SSEA-4 antibodies, and expressed marker genes responsible for maintaining the pluripotent condition. In the cultures of differentiated iPSCs, cells positively stained for the markers of the three primary germ layers (ectoderm, mesoderm, and endoderm) have been revealed. An effective protocol of iPSCs differentiation into dopaminergic neurons has been worked out, and confirmed by the expression of the specific marker tyrosine hydroxylase enzyme.

Conclusion. On the basis of explicitly characterized iPSCs from patients with various forms of PD and the developed cellular protocol, a platform for studying the pathogenesis of PD at the molecular and cellular level has been created. Obtaining cell population enriched with dopaminergic neurons opens a perspective for their application for personalized cell replacement PD therapy.

Key words: cell reprogramming; induced pluripotent stem cells; platform for iPSC; fibroblasts; dopaminergic neurons; Parkinson's disease.

Introduction. The prevalence of Parkinson's disease (PD) is 100-200 cases per 100,000 population, and the portion of the affected persons grows with the age reaching $1-2 \%$ among the people over 60 years $[1,2]$. But in $10 \%$ of patients the disease starts at the young age, i.e. before 40 [3].

For contacts: Sergey N. Illarioshkin, e-mail: snillario@gmail.com 
The main clinical manifestations of PD (bradykinesia, muscular rigidity, rest tremor) are caused by the destruction of dopamine-producing neurons of the midbrain substantia nigra and degeneration of nigrostriate dopaminergic pathway [4]. Genetic factors play an important role in the mechanisms of neurodegenerative process developing in PD. About $10 \%$ of cases are of hereditary character and are associated with mutations of one of 20 known genes of this condition, whereas in the majority of patients a sporadic form of the disease occurs, the development of which being determined by a complex interaction of numerous risk genes and environmental factors [5-7]. The inherited forms of PD develop usually at the young (including adolescent) and middle age, while the sporadic PD form manifests in typical cases in the $6-8^{\text {th }}$ decade of life $[8,9]$.

The most common PD form with autosomal dominant inheritance is PARK8 caused by mutations in the gene of leucine-rich repeat kinase (LRRK2) on chromosome 12q [10]. The debut of the disease after 40 years and formation of Lewy bodies, pathognomonic for PD, are typical for PARK8. Among autosomal recessive cases of PD, of great importance are PARK2, PARK6 and PARK7 forms associated with homozygous or compound heterozygous mutations in PARK2 (6q chromosome), PINK1 and DJ-1 (both loci $-1 \mathrm{p}$ chromosome) genes [10]. The mentioned recessive forms, in aggregate, cause over half of all cases of primary parkinsonism with the onset of symptoms before 20 years of age $[9,11]$. PD gene analysis allowed the researches to determine a number of molecular pathways leading to the progressive loss of dopaminergic neurons. Mitochondrial dysfunction, oxidative and proteolytic stresses have been recognized to be the key factors of PD development [8, 12, 13]; however the exact mechanisms of neurodegeneration of parkinsonian type have not been clarified so far. In this context, PD modeling in vivo and in vitro is of great interest, as it allows scientists to create informative platforms for comprehensive investigation of the pathogenic basis of the condition.

New possibilities in modeling PD and other neurodegenerative disorders were discovered in the second half of 2000s as a result of creating the technology of reprogramming of somatic cell to pluripotent stem cells with their further differentiation into the cells of various types, including neurons [14-19]. Such reprogramming is realized by the introduction of a certain set of transcription factors into the cell (for example, Oct3/4, Sox2, c-Myc and Klf4). The cells obtained in such a way were called by Takahashi and Yamanaka (the authors of the first publication on this topic) induced pluripotent stem cells (iPSCs) [14]. The proliferative potential of iPSCs is actually identical to that of the embryonic stem cells (ESCs). Since iPSCs are able to grow in the culture in vitro for practically unlimited time, investigators obtain the necessary amount of cellular material for carrying out any molecular-biological, neurophysiological and biochemical experiments. To date, sufficiently effective methods of differentiating iPSCs both into the neurons of specific ergicity and into glial cells have been developed [20-23]. Studies of dopaminergic neuron cultures, derived on the basis of iPSCs technology from fibroblasts of PD patients with precisely characterized mutations, will make it possible to clarify fine characteristics of neuron functioning depending on the specific genetic context inherent to one or other patient, and to evaluate the relationships between cytopathologic changes and the clinical syndrome. Comparison of properties of the cellular cultures obtained from the patients with familial (genetic) and sporadic forms of PD is also of great interest.

The aim of the study was to obtain and characterize induced pluripotent stem cells from patients with various forms of Parkinson's disease, and to create on this basis a platform for analyzing the pathogenesis of the disease at the molecular and cellular level, with the development of a protocol of the stem cell differentiation.

\section{Materials and Methods}

Patients. Five patients with PD (4 men and 1 woman) at the age of 58-66 years were included into the study; the age of symptoms debut was 23-57 years. Two patients had autosomal dominant PD form and were heterozygous carriers of G2019S mutation in LRRK2 gene (PARK8 form), one patient had an autosomal recessive form of the disease and was a compound heterozygous carrier of IVS1+1G/A and del202-203(AG) mutations in PARK2 gene (PARK2 form), one patient was a carrier of heterozygous mutation in GBA gene (GBA-associated form), and one patient suffered from a sporadic PD form (Table 1). Skin fibroblasts taken from 3 healthy donors ( 1 man 60 years of age, and 2 women aged 28 and 18 years) served as control samples.

The study complies with the Declaration of Helsinki (the Declaration was passed in Helsinki, Finland, June, 1964, and revised in October, 2000, Edinburg, Scotland) and was performed following approval by the Ethic Committee of the Research Center of Neurology. Written informed consent was obtained from every patient and healthy donors.

Deriving primary cultures of skin fibroblasts. After biopsy, the explant was kept in a medium for skin bioptate (till the delivery to the laboratory) for $1-2 \mathrm{~h}$. The explantat in a drop of medium for fibroblasts was placed on a Petri dish cover $6 \mathrm{~cm}$ in diameter and cut with a sharp scalpel into small pieces (about $0.5 \mathrm{~mm}$ ), each being placed in a separate dish $35 \mathrm{~mm}$ in diameter or by 3-4 pieces in a dish having $6 \mathrm{~cm}$ in diameter, and pressed from above by a sterile cover glass. A medium for fibroblast cultivation was poured on the glass (DMEM, 15\% PBS, $1 \mathrm{mM}$ glutamine, $50 \mathrm{units} / \mathrm{ml}$ penicillin-streptomycin): $4-5 \mathrm{ml}$ of medium on $35 \mathrm{~mm}$ diameter Petri dish, 7-8 $\mathrm{ml}$ on $6 \mathrm{~cm}$ diameter Petri dish. The explantats were incubated in a $\mathrm{CO}_{2}$-incubator in $5 \% \mathrm{CO}_{2}$ and at $37^{\circ} \mathrm{C}$. A week later the cells began to migrate actively from the explants, and the medium was replaced by a fresh one without shifting the cover glass. In about 3 weeks (when a monolayer was formed) the obtained cells were expanded on 1:2 basis. Fibroblasts of the $2-3^{d}$ passage were used for obtaining iPSCs. 
Table 1

General characteristics of the patients-donors with Parkinson disease

\begin{tabular}{lcccll}
\hline $\begin{array}{c}\text { Patient's } \\
\text { code }\end{array}$ & Gender & $\begin{array}{c}\text { Age (years) } \\
\text { when biopsy } \\
\text { was taken }\end{array}$ & $\begin{array}{c}\text { Age (years) } \\
\text { of disease } \\
\text { symptom debut }\end{array}$ & \multicolumn{1}{c}{$\begin{array}{c}\text { Form of the disease, } \\
\text { the character of its course }\end{array}$} & $\begin{array}{c}\text { Genetics } \\
\text { (mutation carriage) }\end{array}$ \\
\hline PDL1 & Male & 58 & 47 & Mixed form, symptom fluctuations, hallucinations & G2019S mutation in LRRK2 gene \\
\hline PDL2 & Male & 63 & 50 & $\begin{array}{l}\text { Akinetic-rigid form, symptom fluctuations, } \\
\text { hallucinations }\end{array}$ & G2019S mutation in LRRK2 gene \\
\hline PDP1 & Male & 64 & 23 & $\begin{array}{l}\text { Akinetic-rigid form, combination } \\
\text { with dystonia, drug-induced dyskinesias }\end{array}$ & $\begin{array}{l}\text { Two mutations in PARK2 gene: deletion } \\
\text { of del202-203AG (exon 2), splicing-mutation } \\
\text { IVS1+1G/A (intron 1) }\end{array}$ \\
\hline PDG1 & Male & 60 & 44 & Mixed form & N370S mutation in GBA gene \\
\hline PDSP1 & Female & 66 & 57 & Mixed form & No mutations (sporadic case) \\
\hline
\end{tabular}

Deriving iPSCs using lentiviral vectors. Human skin fibroblasts were inoculated by 40,000 cells in the medium for fibroblasts with $2 \mathrm{ng} / \mathrm{ml} \mathrm{bFGF}$ on the $35 \mathrm{~mm}$ diameter cultural dish. Two days after expansion the cells were infected with four vectors, obtained previously in our laboratory, for lentiviral expression: LeGO-hOCT4, LeGOhSOX2, LeGO-hc-Myc and LeGO-hKLF4 (MOI - 10, $10,5,5$, respectively) $[16,17]$. Five days after infecting, the cells were reseeded $1: 12$ across the area on the culture dishes with $35 \mathrm{~mm}$ diameter in the medium for fibroblasts. The next day after reseeding this medium was replaced by that for human pluripotent cells. On day 6 after infecting valproic acid was added to the cells up to $1 \mathrm{mM}$ concentration in the medium and BIX-01294 up to its $2 \mu \mathrm{M}$ concentration. Then the cells were cultivated with these "small molecules" for 10-12 days, the medium was changed once in 1-2 days. The low-weight-molecular substances used are inhibitors of histone deacetylase and histone methylase, their application increases several times the efficacy of reprogramming [24, 25]. By day 10-12 numerous colonies of the cells having various morphologies were formed. The colonies morphologically similar to the human embryonal stem cells were mechanically removed and cultured separately in mTeSR1 medium following manufacturer's instructions. A Matrigel substrate (BD Biosciences, USA) was used for culturing.

Deriving iPSCs using Sendai virus vectors. The method described in the manual CytoTune ${ }^{\circledR}$-iPS Reprogramming Kit (https://tools.lifetechnologies.com/ content/sfs/manuals/CytoTune_iPS_Reprogramming Kit_man.pdf) was used to derive iPSCs by integrationfree technique using Sendai virus, carrying in its genome transcription factors Oct4, Sox2, KIf4 and c-Myc. The day before the transfection 300,000 human skin fibroblasts were inoculated on a well of 6 -well plate in $2 \mathrm{ml}$ medium for fibroblast cultivation. The next day, transduction of the cells with viruses in an amount of 5 viral particles per 1 cell (MOI5) was performed. The cells were incubated with the viral particles for $24 \mathrm{~h}$ in a $\mathrm{CO}_{2}$-incubator in $5 \%$ $\mathrm{CO}_{2}$ and at $37^{\circ} \mathrm{C}$. Then the medium was changed for a fresh one every other day. Five days later the infected cells were reseeded $(50,000$ cells per Petri dish $10 \mathrm{~cm}$ in diameter) using $0.25 \%$ trypsin solution on the feeder layer from the human foreskin fibroblasts, treated with mitomycin $\mathrm{C}$. From this moment the cells were cultivated in the medium for pluripotent human cells containing $0.5 \mathrm{mM}$ sodium butyrate.

On day 20 after inoculation clones were selected according to the morphological criterion of similarity with the colonies of human ESC. The selected clones were then cultivated as separate lines in mTeSR1 medium on a matrigel substance.

Immunocytochemistry. The cells on the Petri dish, prepared for staining, were washed twice in PBS, fixed with $4 \%$ paraformaldehyde in PBS (pH 6.8) for 20 min at the room temperature, washed three times in PBS with $0.1 \%$ Tween 20. Cell membranes were permeabilized with PBS solution with $0.1 \%$ Triton X100 for 10 min at the room temperature. Nonspecific sorption of antibodies was blocked by incubation for $30 \mathrm{~min}$ in PBS with $0.1 \%$ Tween 20 (5\% PBS, $2 \%$ goat serum) at the room temperature. Antibodies against the following markers were used in the work: Oct4, Nanog, SSEA4, Tra-181 (Cell Signaling, USA), CD105 (DAKO, Denmark), pancytokeratine (Thermo Scientific, USA), $\beta$-III-tubulin, a-fetoprotein, tyrosine hydroxylase, tropomyosin (Abcam, USA). Primary antibodies (Invitrogen, USA) were applied in dilutions recommended by the manufacturer: surface antigens were incubated for $1 \mathrm{~h}$ at the room temperature in PBS with $0.1 \%$ Tween 20 (5\% PBS, $2 \%$ goat serum), cytoplasmic and nuclear antigens were incubated overnight at $+4^{\circ} \mathrm{C}$, then all antibodies were washed 3 times for 5 min in PBS with $0.1 \%$ Tween 20 . The secondary antibodies (Invitrogen, USA) conjugated with fluorescent labels (Alexa 488, Alexa 555, Alexa 546, Alexa 647) were applied in dilutions recommended by the manufacturer: they were incubated for $30 \mathrm{~min}$ at the room temperature in the dark, washed 3 times for 5 min in PBS with $0.1 \%$ Tween 20 . Then the preparations were incubated with $0.1 \mathrm{\mu g} / \mathrm{ml}$ DAPI (4',6-diamidino2-phenylindole dihydrochloride) in PBS for $10 \mathrm{~min}$ for visualization of the cell nuclei, and washed twice in PBS, 
Table 2

Primers and conditions of their amplification

\begin{tabular}{|c|c|c|}
\hline Gene & Primer sequences & Annealing conditions \\
\hline OCT4 & $\begin{array}{l}\text { 5'CGACCATCTGCCGCTTTGAG3' } \\
\text { 5'CCCCCTGTCCCCCATTCCTA3' }\end{array}$ & $69^{\circ} \mathrm{C}, 30$ cycles \\
\hline SOX2 & $\begin{array}{l}\text { 5'TCCTGATTCCAGTTTGCCTC3' } \\
\text { 5'GCTTAGCCTCGTCGATGAAC3' }\end{array}$ & $69^{\circ} \mathrm{C}, 30$ cycles \\
\hline NANOG & $\begin{array}{l}\text { 5'CAGCCCTGATTCTTCCACCAGTCCC3' } \\
\text { 5'TGGAAGGTTCCCAGTCGGGTTCACC3' }\end{array}$ & $69^{\circ} \mathrm{C}, 30$ cycles \\
\hline DPPA4 & $\begin{array}{l}\text { 5'CAAGCCCAAGAACAGCCTAGTGAA3' } \\
\text { 5'TGACGAAGCAGTCGTTGAGTGAGA3' }\end{array}$ & $63^{\circ} \mathrm{C}, 30$ cycles \\
\hline HESX1 & $\begin{array}{l}\text { 5'ACCTGCAGCTCATCAGGGAAAGAT3' } \\
\text { 5'AAAGCAGTTCTTGGTCTCGGCCT3' }\end{array}$ & $66^{\circ} \mathrm{C}, 30$ cycles \\
\hline TH & $\begin{array}{l}\text { 5' CCAAGCAGGCAGAGGCCATCATGT3' } \\
\text { 5' GGCGTAGAGGCCCTTCAGCGT3' }\end{array}$ & $60^{\circ} \mathrm{C}, 30$ cycles \\
\hline SYN & $\begin{array}{l}\text { 5' GCTTTGTGAAGGTGCTGCAA3' } \\
\text { 5'GCCTGAAGGGGTACTCGAAC3' }\end{array}$ & $60^{\circ} \mathrm{C}, 30$ cycles \\
\hline GAPDH & $\begin{array}{l}\text { 5'GAAGGTGAAGGTCGGAGTCA3' } \\
\text { 5'TTCACACCCATGACGAACAT3' }\end{array}$ & $60^{\circ} \mathrm{C}, 25$ cycles \\
\hline
\end{tabular}

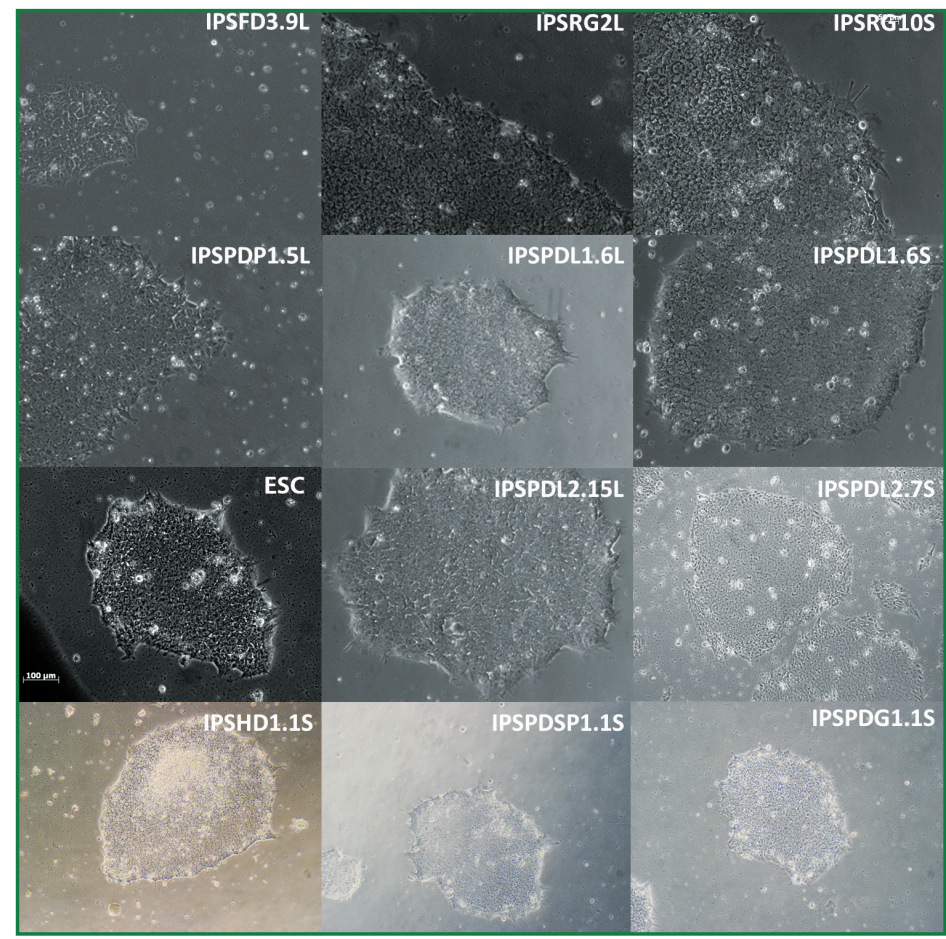

Figure 1. Morphology of iPSCs derived by various methods. Here: IPSRG2L and IPSRG10S are iPSCs lines obtained from healthy donor 1 with the help of lentivirus and Sendai virus, respectively; IPSHD1 and IPSFD3 are iPSCs lines obtained from healthy donor 2 and 3 with the help of Sendai virus and lentivirus, respectively; IPSPDL1 (L2), IPSPDP1 and IPSPDG1 are IPSCs lines obtained from patients with $P D$ - carriers of mutations in genes LRRK2, PARK2 and GBA, respectively; IPSPDSP1 is iPSCs line from the patient with a sporadic form of PD. In the names of the clones figures with letters $L$ and $S$ indicated after a point designate the number of the clones derived by means of integration (lentiviral infection, L) or integration-free (infection by Sendai virus, S) methods, respectively. ESC means embryonal stem cells. $\times 100$ after that they were examined under a fluorescence microscope (LSM 510; Zeiss, Germany).

Reverse transcription polymerase chain reaction (RT-PCR). A total RNA from the cell culture was isolated with the help of RNeasy mini kit according to the manufacturer's instruction. Treatment with DNase was performed directly on the columns using RNaseFree DNase Set (QIAGEN, USA). RNA concentration was determined by Qubit following the manufacturer's instructions. Reverse transcription reaction was performed using random 6-nucleotide primers, MMLV reverse transcriptase, inhibitors of ribonucleases and deoxyribonucleotides according to the manufacturer's instruction. 1-2 $\mu \mathrm{g}$ of total RNA was taken per one reaction. PCR-amplification of reaction products was conducted with the help of Taq polymerase (as indicated in manufacturer's instructions) in the amplificator (Eppendorf, USA). The list of primers used in the work and amplification conditions are shown in Table 2. PCR-products were analyzed by means of electrophoresis in 1-2\% agarose gel.

Formation and cultivation of embryoid bodies. The iPSCs colonies were removed with $1 \mathrm{mg} /$ $\mathrm{ml}$ dispase, dissociated into the fragments of 400-600 cells, and transferred in the mTeSR1 medium to a well of the 24-well plate (Ultra Low Adhesion Plates, Corning, USA). The next day half the medium was replaced by that for culturing embryoid bodies. Then mTeSR1 was gradually changed for the medium for embryoid bodies (DMEM/F12, 20\% PBS, 2 mM glutamine, $0.1 \mathrm{mM}$ $\beta$-mercaptoethanol, $1 \%$ mixture of essential amino acids, 50 units $/ \mathrm{ml}$ penicillin-streptomycin; $50 \mu \mathrm{g} /$ $\mathrm{ml}$ ). The medium was changed once every 2 days, adding $2 \mathrm{ml}$ medium in each well.

Spontaneous differentiation of human iPSCs. Embryoid bodies at the age of 10-20 days were seeded on Petri dishes covered with $0.1 \%$ gelatin in the medium designed for the given bodies. The embryoid bodies attached to the gelatin substrate, and migration of the cells to the dish surface began. 2-3 weeks later vast regions of differentiated cells were formed and stained with antibodies for the markers of the derivatives of the 3 germ layers (ectoderm, mesoderm, entoderm).

\section{Results}

iPSCs obtained by integration and integration-free techniques and the characteristic of the cells derived from healthy donors and patients with PD. Skin bioptates taken from PD patients and healthy donors were used for isolation of primary fibroblast cultures and then for obtaining iPSCs with the help of lentiviral transfection (integration technique) and transduction by Sendai virus (integration-free technique).

ESC-like colonies began to emerge 14 days after infecting the cell lines with lentiviruses. On day 20-25 the grown colonies were mechanically 
transferred in mTeSR medium on the Matrigel substrate (BD Biosciences, USA). About 100 clones of ESC-like colonies were generated from 40,000 fibroblasts taken to be infected with lentiviruses carrying "Yamanaka cocktail" genes, i.e. the efficacy of reprogramming amounted to $0.2 \%$. The efficacy of deriving iPSCs using Sendai virus appeared to be similar to that of using lentiviral constructions (about $0.2 \%$ ), but the colonies morphologically similar to ESC started to appear earlier already on day 10 after infecting (Figure 1).

The derived iPSCs clones were morphologically similar to human ESCs: they were about $20 \mu \mathrm{m}$ in size, had a large nucleus/cytoplasm ratio, and grew by monolayer colonies in a tight contact with adjacent cells (See Figure 1). Some iPSCs lines, obtained by using Sendai virus, were prone to form colonies of irregular configuration (angular or stellate), which were not observed in the iPSCs lines after lentiviral infecting.

A cytogenetic analysis of iPSCs clones, derived by GTG-differential staining, showed that all of them had normal 46XY karyotype.

In the process of reprogramming to pluripotent state significant alterations in the pattern of gene expression took place. Genes appropriate to the differentiated state of the cell became "silent", whereas genes maintaining pluripotent state were activated. Expression in iPSCs

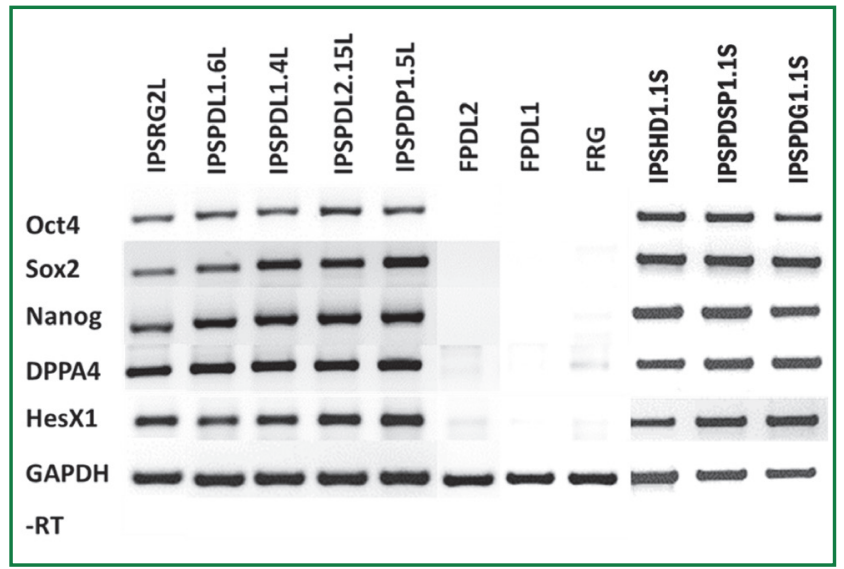

Figure 2. Comparison of expression of the genes typical for pluripotent state (Oct4, Sox2, Nanog, DPPA4, HesX1, GAPDH, -RT) in the obtained iPSCs lines, initial lines of human skin fibroblasts and ESCs by RT-PCR method. Here: IPSRG2L is iPSCs line derived from healthy donor 1 with the help of lentivirus; IPSHD1 is iPSCs line derived from healthy donor 2 with the help of Sendai virus; IPSPDL1 (L2), IPSPDP1 and IPSPDG1 are iPSCs lines derived from patients with $P D$ - carries of mutations in genes $L R R K 2$, PARK2 and GBA, respectively; IPSPDSP1 is iPSCs line from a patent with a sporadic form of PD. In the names of the clones figures with letters $L$ and $S$ indicated after a point designate the number of the clones derived by means of integration (lentiviral infection, L) or integration-free (infection by Sendai virus, S) methods, respectively. FPDL2, FPDL and RRG are fibroblast lines. $\times 100$

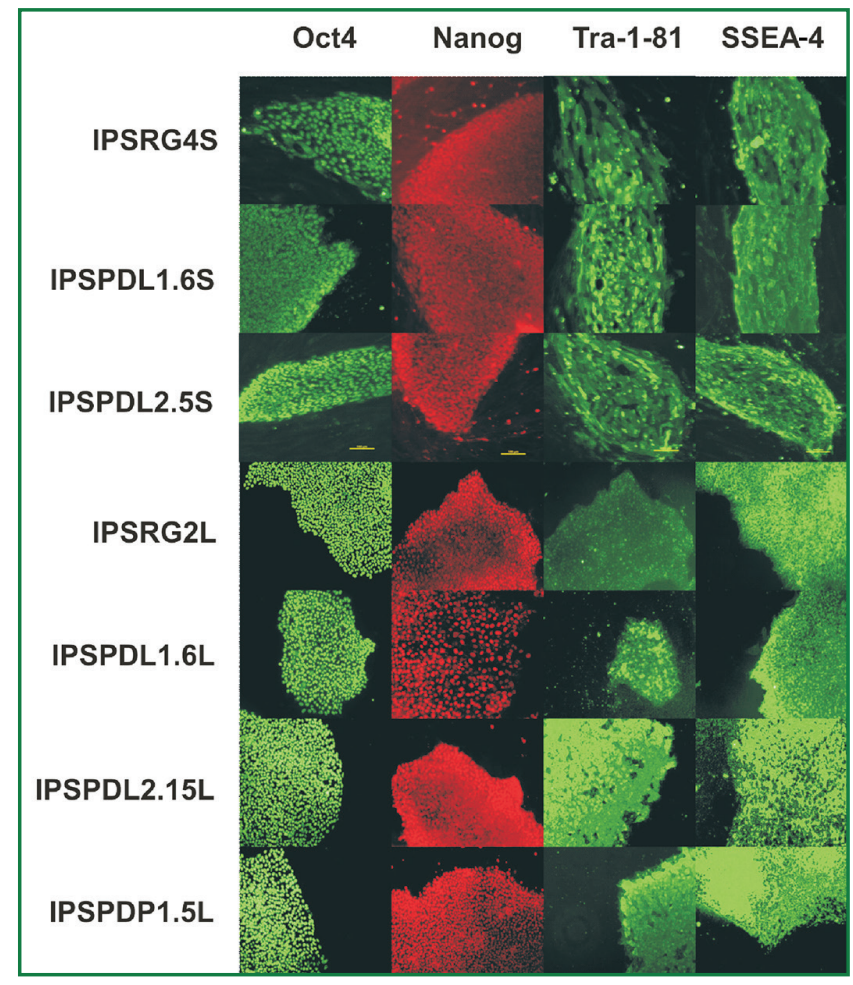

Figure 3. Immunocytochemical analysis of pluripotency marker expression: Oct4, Sox2, Nanog, Tra-1-81 and SSEA-4 in iPSCs lines. Here: IPSRG2L and IPSRG4S are iPSCs lines obtained from healthy donor 1 with the help of lentivirus and Sendai virus, respectively; IPSPDL1 (L2), IPSPDP1 are iPSCs lines obtained from patients with PD - carriers of mutations in genes $L R R K 2$ and PARK2, respectively. In the names of the clones figures with letters $L$ and $S$ indicated after a point designate the number of the clones derived by means of integration (lentiviral infection, L) or integration-free (infection by Sendai virus, S) methods, respectively. $\times 100$

clones of the marker genes responsible for maintaining pluripotent state is shown on Figure 2.

The derived iPSCs lines were analyzed for specific markers with the help of antibodies. All clones selected for the analysis were specifically stained by antibodies for Oct4, Nanog, TRA-1-81 and SSEA-4 (Figure 3).

To confirm functionally the pluripotent status of the iPSCs clones, the test for forming embryoid bodies by the cells, as well as the analysis of their further differentiation into the cells belonging to the three germ layers were performed. It was demonstrated, that in the cultures of spontaneously differentiated iPSCs there were cells being positively stained for the markers of ectoderm (pancytokeratine, $\beta$-III-tubulin), mesoderm (CD105, tropomyosin) and entoderm ( $\alpha$-fetoprotein) (Figure 4). The capacity for spontaneous differentiation did not depend on the method of deriving iPSCs lines.

Protocol of differentiating iPSCs into dopaminergic neurons. A protocol published by Friks et al. in 2011, revised and adapted for our conditions, was used for 
directed differentiation [26]. Following this protocol we failed to reach the claimed efficacy of differentiation in $80 \%$ of TH-positive neurons. Application of cultural media and growth factors of other manufacturers was likely to be the reason of such a result. Besides, in the mentioned protocol iPSCs differentiation began after additional growing of the cells in the conditioned medium from the mouse embryonal fibroblasts. We excluded this stage, connected with the components of the animal origin and uncertain composition; therefore, prior to differentiation, iPSCs were additionally grown in the commercially available mTeSR1 medium for pluripotent human cells, which required changes in the incubation time with various differentiation factors. We also introduced an additional stage of reseeding and/or freezing neuronal progenitors, which allowed us to preserve them at different stages of differentiation and to save expensive protein growth factors.

The protocol of differentiation consisted of three stages. Prior to differentiation human iPSCs were cultivated until reaching $70-80 \%$ monolayer in mTeSR1 medium on Matrigel substrate. Induction of neuronal differentiation was based on dual inhibition of SMAD signal pathway [27], therefore, at the first stage the medium was changed for the medium designed for neuronal differentiation containing $80 \mathrm{ng} / \mathrm{ml}$ Noggin, $10 \mu \mathrm{M}$ SB431542, $4 \mu \mathrm{M}$ dorsomorphin. Low-molecularweight dorsomorphin is an inhibitor of the BMP pathway [28]. Using a set of inductors of neural differentiation, we succeeded in obtaining practically pure population of neuronal progenitors phenotypically corresponding to the cells of the neural tube. At this stage general neuronal

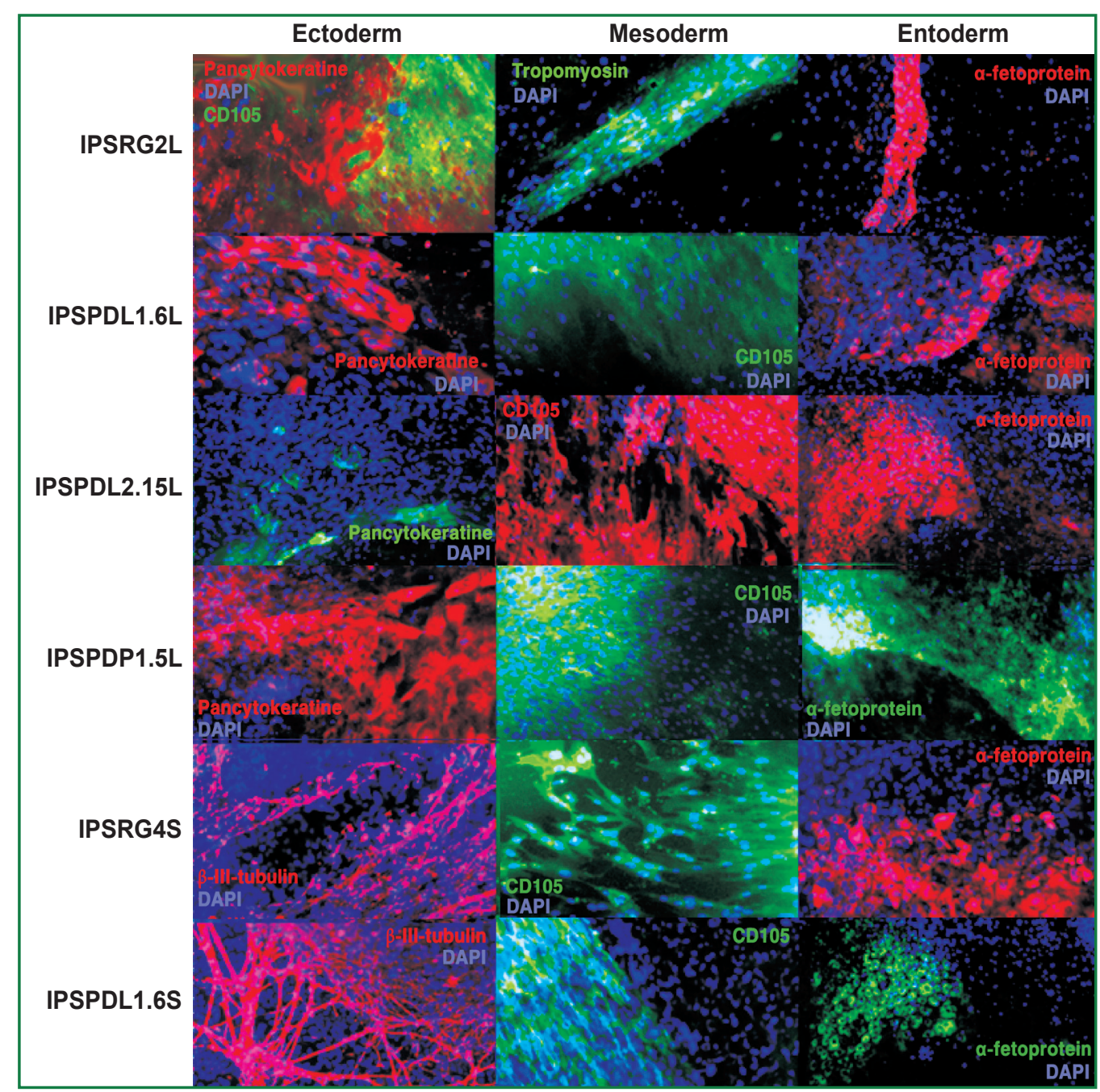

Figure 4. Immunocytochemical analysis of differentiated iPSCs derivatives for the ability to form cell derivatives of the three germ layers. Shown are markers of ectoderm (pancytokeratine, $\beta$-III-tubulin and NeuN), mesoderm (CD105, tropomyosin and desmin) and entoderm (a-fetoprotein). Here: IPSRG2L and IPSRG4S are iPSCs lines obtained from healthy donor 1 with the help of lentivirus and Sendai virus, respectively; IPSPDL1 (L2) and IPSPDP1 are iPSCs lines obtained from patients with $P D$ - carriers of mutations in genes $L R R K 2$ and PARK2, respectively. In the names of the clones figures with letters $L$ and $S$ indicated after a point designate the number of the clones derived by means of integration (lentiviral infection, $L$ ) or integration-free (infection by Sendai virus, S) methods, respectively. $\times 100$ 
Figure 5. Analysis of neuronal cultures on day 38 of differentiation for the expression of neuronal markers and markers of the neuron mature state by reverse transcription polymerase chain reaction (RT-PCR) and immunocytochemistry. (a) PCR-analysis of neural cultures differentiated from iPSCs lines on day 34 and 54 of differentiation. Here: IPSRG2L is iPSCs line derived from healthy donor 1 with the help of lentivirus; IPSPDL1 (L2) are iPSCs lines obtained from patients with $P D$ - carriers of mutations in genes LRRK2. In the names of the clones figures with letters $L$ and $S$ indicated after a point designate the number of the clones derived by means of integration (lentiviral infection, L) or integration-free (infection by Sendai virus, S) methods, respectively. TH is tyrosine hydroxylase, SYN - synaptophysin, GAPDH glyceraldehyde phosphate dehydrogenase. (b) Immunocytochemical analysis of neuronally differentiated iPSCs derivatives. $\times 100$
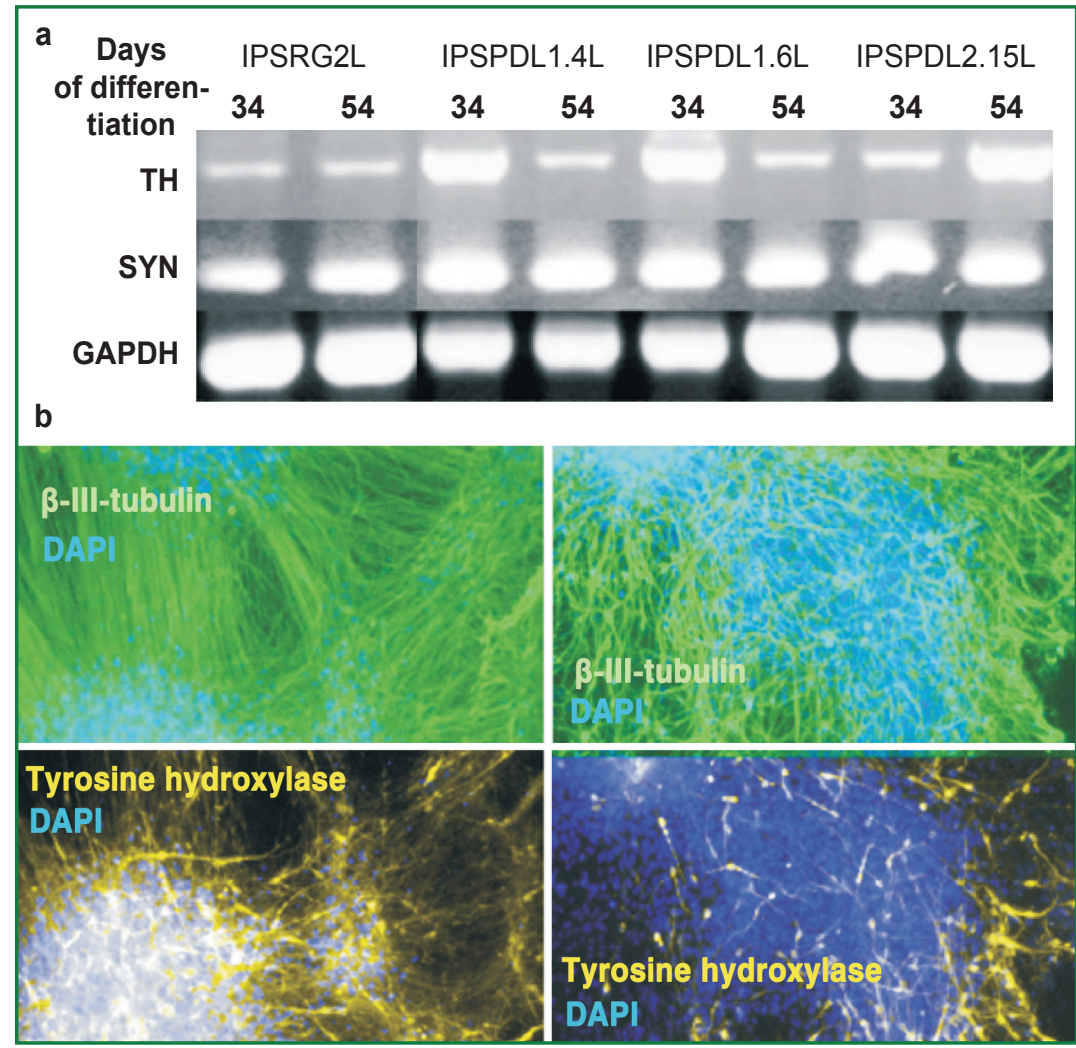

progenitors are formed, which are capable in case of further differentiation to give all types of neuronal and glial cells. Such progenitors easily endure freezing, and can be stored in liquid nitrogen. The efficacy of obtaining neuronal progenitors is very high: from (1-2). $10^{6}$ iPSCs involved in differentiation about $10 \cdot 10^{6}$ progenitor cells are successfully derived after 14 days of the first stage of neuronal differentiation.

Sonic hedgehog (Shh) factor and fibroblast growth factor 8 (FGF8) are responsible for the specialization of dopaminergic neurons of the midbrain substantia nigra [29, 30]. For this reason, high concentrations of Shh and FGF8, 200 and $100 \mathrm{ng} / \mathrm{ml}$, respectively, were used for specialization of neuron progenitors obtained at the first stage. At the second stage, Shh in the culture medium can be partly replaced by a lowmolecular-weight purmorphamin [31]. In this case the concentration of recombinant Shh in the culture medium may be decreased to $100 \mathrm{ng} / \mathrm{ml}$, which is economically profitable. After the completion of this stage, neuronal progenitors change morphologically, the body of the cell and the process, the length of which does not exceed three diameters of the cell body, can be seen. Such cells are already the progenitors of the midbrain neurons and may be reseeded by Versen solution and subject to conservation. The possibility to preserve neuron progenitors in liquid nitrogen at various differentiation stages allows us to create the bank of such differentiated derivatives. It eliminates the necessity to start differentiation each time from the stage of iPSCs when making the experiments with mature neurons, saving time and money.

At the third stage of differentiation, the cells were cultivated in the presence of BDNF and GDNF factors, promoting neuron maturation. Forskolin was also present in the medium for culturing, providing selective survival of postmitotic cells and decreasing, therefore, the number of glial cells [32]. Ascorbic acid was used as an antioxidant. Neuronal differentiation according to the developed protocol takes about 40 days, and thereafter the cells may be maintained in the medium for maturation in the presence of BDNF, GDNF and ascorbic acid at least 40 days more. Differentiated cells were shown to express a characteristic marker for mature neurons synaptophysin (Figure $5(\mathrm{a})$ ), participating in the release of neuromediator [33]. The cultures of neurons obtained by us expressed also the marker of catecholaminergic (dopaminergic, in particular) neurons - tyrosine hydroxylase (Figure 5 (b)).

Thus, as a result of the investigations performed, an effective method of deriving the cell population enriched with dopaminergic neurons has been developed. These cell cultures may be applied both for further studies of the mechanisms of PD development and for screening potential drugs with neuroprotective activity.

Discussion. The development of somatic cell reprogramming technology and obtaining mammalian and human iPSCs open new perspectives in transplantology and investigation of molecular and cellular basics of severe humans diseases in vitro [4, 34]. Moreover, further 
development of this technology gives the opportunity for creating the models of numerous severe human pathologies, including neurodegenerative [23], and test systems enabling researchers to carry out an extensive screening and clarify the properties of pharmaceuticles directed to the treatment of particular diseases in vitro, considering individual characteristics of the patient. The fact that the reprogramming technology makes it possible to derive iPSCs from individual differentiated somatic cells of the affected and healthy donors gives it significant advantages over ESCs technology, which, in its turn, opens wide perspectives for the development of personalized medicine. Further improvement of such technology will diminish the number of the experiments for testing promising pharmacological compounds on animal cells, performing them directly of the human cells. The possibility to differentiate iPSCs into cardiomyocytes, hepatocytes, fibroblasts, neurons and other cells will allow scientists to conduct a targeted preclinical toxicological screening of the compounds in vitro.

iPSCs derived by means of various methods, especially those not involving the gene structure in the somatic cells (virus-free reprogramming methods), may be applied in the cellular therapy of severe human disorders, when it is necessary to restore the cells and organs damaged by some pathological process or trauma. With the help of iPSCs it is also possible to correct congenital mutations in the genes responsible for various hereditary illnesses. In recent years there appeared some works $[35,36]$ devoted to this important problem, and their number is sure to increase. However, a wide application of iPSCs for cellular therapy is limited so far due to some reasons, the main being their possible malignization in the organism. Besides, an exceptional complexity of the human nervous system organization poses additional problems for using iPSCs in neurotransplantation. Firstly, it is obtaining the neurons of certain ergicity in sufficient quantities and providing their viability after transplantation. Secondly, the transplanted cells should be correctly built in certain brain areas including their differentiation and establishment of interneuronal contacts. And finally, it is important to provide functional activity of transplanted iPSCs. Despite all these difficulties, rapidly developing technologies of cellular reprogramming are at present among most perspective in the molecular medicine [37].

Conclusion. A platform for studying the pathogenesis of PD at the molecular and cellular level has been created on the basis of explicitly characterized iPSCs from patients with various forms of $\mathrm{PD}$ and the developed cell protocol. Obtaining a sufficient quantity of cell population enriched with dopaminergic neurons opens a perspective for their application for personalized cell replacement PD therapy.

Acknowledgement. The authors thank the Center of Collective Use of Scientific Equipment of the Institute of Molecular Genetics of the Russian Academy of Sciences "The Center of Cellular and Gene Technology", whose equipment was used in the study.
Study Funding. The work was supported by grant No.14-15-01047 of the Russian Science Foundation.

Conflicts of Interest. The authors have no conflicts of interest.

\section{References}

1. Kasten M., Chade A., Tanner C.M. Epidemiology of Parkinson's disease. In: Handbook of clinical neurology. Part I. Elsevier; 2007; p. 129-151, https://doi.org/10.1016/s00729752(07)83006-5.

2. Wirdefeldt K., Adami H.-O., Cole P., Trichopoulos D., Mandel J. Epidemiology and etiology of Parkinson's disease: a review of the evidence. Eur J Epidemiol 2011; 26(S1): 1-58, https://doi.org/10.1007/s10654-011-9581-6.

3. Illarioshkin S.N. Parkinsonism with an early onset. Nervnye bolezni 2006; 3: 14-20.

4. Thomas B., Beal M.F. Parkinson's disease. Hum Mol Genet 2007; 16: R183-R194, https://doi.org/10.1093/hmg/ ddm159.

5. Illarioshkin S.N. DNK-diagnostika i medikogeneticheskoe konsul'tirovanie [DNA diagnostics and genetic consultation]. Moscow: MIA; 2004.

6. llarioshkin S.N. Modern concepts of Parkinson's disease etiology. Nevrologicheskiy zhurnal 2015; 20(4): 4-13.

7. Lin M.K., Farrer M.J. Genetics and genomics of Parkinson's disease. Genome Med 2014; 6(6): 48, https://doi. org/10.1186/gm566.

8. Schiesling C., Kieper N., Seidel K., Krüger R. Review: familial Parkinson's disease - genetics, clinical phenotype and neuropathology in relation to the common sporadic form of the disease. Neuropathol Appl Neurobiol 2008; 34(3): 255271, https://doi.org/10.1111/j.1365-2990.2008.00952.x.

9. Chai C., Lim K.L. Genetic insights into sporadic Parkinson's disease pathogenesis. Curr Genomics 2013; 14(8): 486-501, https://doi.org/10.2174/1389202914666131210195808.

10. Healy D.G., Falchi M., O'Sullivan S.S., Bonifati V., Durr A., Bressman S., Brice A., Aasly J., Zabetian C.P., Goldwurm S., Ferreira J.J., Tolosa E., Kay D.M., Klein C., Williams D.R., Marras C., Lang A.E., Wszolek Z.K., Berciano J., Schapira A.H., Lynch T., Bhatia K.P., Gasser T., Lees A.J., Wood N.W.; International LRRK2 Consortium. Phenotype, genotype, and worldwide genetic penetrance of LRRK2-associated Parkinson's disease: a case-control study. Lancet Neurol 2008; 7(7): 583-590, https://doi.org/10.1016/ S1474-4422(08)70117-0.

11. Kilarski L.L., Pearson J.P., Newsway V., Majounie E., Knipe M.D., Misbahuddin A., Chinnery P.F., Burn D.J., Clarke C.E., Marion M.H., Lewthwaite A.J., Nicholl D.J., Wood N.W., Morrison K.E., Williams-Gray C.H., Evans J.R., Sawcer S.J., Barker R.A., Wickremaratchi M.M., BenShlomo Y., Williams N.M., Morris H.R. Systematic review and UK-based study of PARK2 (parkin), PINK1, PARK7 (DJ-1) and LRRK2 in early-onset Parkinson's disease. Mov Disord 2012; 27(12): 1522-1529, https://doi.org/10.1002/mds.25132.

12. Saiki S., Sato S., Hattori N. Molecular pathogenesis of Parkinson's disease: update. J Neurol Neurosurg Psychiatry 2012; 83(4): 430-436, https://doi.org/10.1136/jnnp-2011-301205.

13. Sulzer D., Surmeier D.J. Neuronal vulnerability, pathogenesis, and Parkinson's disease. Mov Disord 2013; 28(1): 41-50, https://doi.org/10.1002/mds.25095.

14. Takahashi K., Yamanaka S. Induction of pluripotent stem cells from mouse embryonic and adult fibroblast cultures 
by defined factors. Cell 2006; 126(4): 663-676, https://doi. org/10.1016/j.cell.2006.07.024.

15. Nakagawa M., Koyanagi M., Tanabe K., Takahashi K., Ichisaka T., Aoi T., Okita K., Mochiduki Y., Takizawa N., Yamanaka S. Generation of induced pluripotent stem cells without Myc from mouse and human fibroblasts. Nat Biotechnol 2008; 26(1): 101-106, https://doi.org/10.1038/ nbt1374.

16. Nekrasov E.D., Lebedeva O.S., Chestkov I.V., Syusina M.A., Fedotova E.Yu., Lagarkova M.A., Kiselev S.L., Grivennikov I.A., Illarioshkin S.N. Obtaining and characteristics of human induced pluripotent stem cells from skin fibroblasts of patients with neurodegenerative diseases. Geny i kletki 2011; 6(4): 82-88.

17. Lebedeva O.S., Lagar'kova M.A., Kiselev S.L., Mukhina I.V., Vedunova M.V., Usova O.V., Stavrovskaya A.V., Yamshchikova N.G., Fedotova E.Yu., Grivennikov I.A., Khaspekov L.G., Illarioshkin S.N. The morphofunctional properties of induced pluripotent stem cells derived from human skin fibroblasts and differentiated to dopaminergic neurons. Neurochemical Journal 2013; 7(3): 207-214, https:// doi.org/10.1134/s1819712413030082.

18. Medvedev S.P., Shevchenko A.I., Sukhikh G.T., Zakiyan S.M. Indutsirovannye plyuripotentnye stvolovye kletki [Induced pluripotent stem cells]. Novosibirsk: Izd-vo SO RAN; 2014.

19. Bogomazova A.N., Vassina E.M., Kiselev S.L., Lagarkova M.A., Lebedeva O.S., Nekrasov E.D., Panova A.V., Philonenko E.S., Khomyakova E.A., Tskhovrebova L.V., Chestkov I.V., Shutova M.V. Genetic cell reprogramming: a new technology for basic research and applied usage. Russian Journal of Genetics 2015; 51(4): 386-396, https://doi. org/10.1134/s102279541504002x.

20. Byers B., Lee H., Reijo Pera R. Modeling Parkinson's disease using induced pluripotent stem cells. Curr Neurol Neurosci Rep 2012; 12(3): 237-242, https://doi.org/10.1007/ s11910-012-0270-y.

21. Yoshikawa T., Samata B., Ogura A., Miyamoto S., Takahashi J. Systemic administration of valproic acid and zonisamide promotes differentiation of induced pluripotent stem cell-derived dopaminergic neurons. Front Cell Neurosci 2013; 7, https://doi.org/10.3389/fncel.2013.00011.

22. Narytnyk A., Verdon B., Loughney A., Sweeney M., Clewes O., Taggart M.J., Sieber-Blum M. Differentiation of human epidermal neural crest stem cells (hEPI-NCSC) into virtually homogenous populations of dopaminergic neurons. Stem Cell Rev 2014; 10(2): 316-326, https://doi.org/10.1007/ s12015-013-9493-9.

23. Nekrasov E.D., Vigont V.A., Klyushnikov S.A., Lebedeva O.S., Vassina E.M., Bogomazova A.N., Chestkov I.V., Semashko T.A., Kiseleva E., Suldina L.A., Bobrovsky P.A., Zimina O.A., Ryazantseva M.A., Skopin A.Y., Illarioshkin S.N., Kaznacheyeva E.V., Lagarkova M.A., Kiselev S.L. Manifestation of Huntington's disease pathology in human induced pluripotent stem cell-derived neurons. Mol Neurodegener 2016; 11: 27 , https://doi.org/10.1186/s13024-016-0092-5.

24. Huangfu D., Maehr R., Guo W., Eijkelenboom A., Snitow M., Chen A.E., Melton D.A. Induction of pluripotent stem cells by defined factors is greatly improved by smallmolecule compounds. Nat Biotechnol 2008; 26(7): 795-797, https://doi.org/10.1038/nbt1418.

25. Shi Y., Do J.T., Desponts C., Hahm H.S., Schöler H.R., Ding S. A combined chemical and genetic approach for the generation of induced pluripotent stem cells. Cell Stem Cell 2008; 2(6): 525-528, https://doi.org/10.1016/j. stem.2008.05.011.

26. Kriks S., Shim J.W., Piao J., Ganat Y.M., Wakeman D.R., Xie Z., Carrillo-Reid L., Auyeung G., Antonacci C., Buch A., Yang L., Beal M.F., Surmeier D.J., Kordower J.H., Tabar V., Studer L. Dopamine neurons derived from human ES cells efficiently engraft in animal models of Parkinson's disease. Nature 2011; 480(7378): 547-551, https://doi.org/10.1038/nature10648.

27. Chambers S.M., Fasano C.A., Papapetrou E.P., Tomishima M., Sadelain M., Studer L. Highly efficient neural conversion of human ES and iPS cells by dual inhibition of SMAD signaling. Nat Biotechnol 2009; 27(3): 275-280, https:// doi.org/10.1038/nbt.1529.

28. Yu P.B., Hong C.C., Sachidanandan C., Babitt J.L., Deng D.Y., Hoyng S.A., Lin H.Y., Bloch K.D., Peterson R.T. Dorsomorphin inhibits BMP signals required for embryogenesis and iron metabolism. Nat Chem Biol 2008; 4(1): 33-41, https:// doi.org/10.1038/nchembio.2007.54.

29. Gilbert S.F. Developmental biology. Sunderland: Sinauer Associates; 2003.

30. Wurst W., Bally-Cuif L. Neural plate patterning: upstream and downstream of the isthmic organizer. Nat Rev Neurosci 2001; 2(2): 99-108, https://doi.org/10.1038/35053516.

31. Li X.-J., Hu B.-Y., Jones S.A., Zhang Y.-S., LaVaute T., Du Z.-W., Zhang S.-C. Directed differentiation of ventral spinal progenitors and motor neurons from human embryonic stem cells by small molecules. Stem Cells 2008; 26(4): 886-893, https://doi.org/10.1634/stemcells.2007-0620.

32. Hochbaum D., Barila G., Ribeiro-Neto F., Altschuler D.L. Radixin assembles cAMP effectors Epac and PKA into a functional CAMP compartment: role in CAMP-dependent cell proliferation. J Biol Chem 2010; 286(1): 859-866, https://doi. org/10.1074/jbc.m110.163816.

33. Sarnat H.B. Clinical neuropathology practice guide 5-2013: markers of neuronal maturation. Clin Neuropathol 2013; 32(9): 340-369, https://doi.org/10.5414/np300638.

34. Cooper O., Seo H., Andrabi S., Guardia-Laguarta C., Graziotto J., Sundberg M., McLean J.R., Carrillo-Reid L., Xie Z., Osborn T., Hargus G., Deleidi M., Lawson T., Bogetofte H., Perez-Torres E., Clark L., Moskowitz C., Mazzulli J., Chen L., Volpicelli-Daley L., Romero N., Jiang H., Uitti R.J., Huang Z., Opala G., Scarffe L.A., Dawson V.L., Klein C., Feng J., Ross O.A., Trojanowski J.Q., Lee V.M. Marder K., Surmeier D.J., Wszolek Z.K., Przedborski S., Krainc D., Dawson T.M., Isacson O. Pharmacological rescue of mitochondrial deficits in iPSC-derived neural cells from patients with familial Parkinson's disease. Sci Trans/ Med 2012, 4(141): 141ra90, https://doi.org/10.1126/scitranslmed.3003985.

35. Howden S.E., Thomson J.A. Gene targeting of human pluripotent stem cells by homologous recombination. Methods Mol Biol 2014; 1114: 37-55, https:Ildol.org/10.1007/978-162703-761-7_4.

36. Mukherjee S., Thrasher A.J. Gene correction of induced pluripotent stem cells derived from a murine model of $\mathrm{X}$-linked chronic granulomatous disorder. Methods Mol Biol 2014; 1114: 427-440, https://doi.org/10.1007/978-1-62703-761-7_28.

37. Rao M., Gottesfeld J.M. Introduction to thematic minireview series: development of human therapeutics based on induced pluripotent stem cell (iPSC) technology. J Biol Chem 2013, 289(8): 4553-4554, https://doi.org/10.1074/jbc. r113.543652. 